\title{
THE TWO-ENVELOPE PROBLEM REVISITED
}

\author{
DEREK ABBOTT* and BRUCE R. DAVIS ${ }^{\dagger}$ \\ School of Electrical \& Electronic Engineering \\ University of Adelaide, Adelaide, SA 5005, Australia \\ *dabbott@eleceng.adelaide.edu.au \\ †davis@eleceng.adelaide.edu.au \\ JUAN M. R. PARRONDO \\ Departamento Física Atómica, Molecular y Nuclear \\ Universidad Complutense de Madrid, 28040-Madrid, Spain \\ parrondo@fis.ucm.es \\ Received 18 June 2009 \\ Accepted 17 September 2009 \\ Communicated by Natalia B. Janson
}

\begin{abstract}
The two-envelope problem has intrigued mathematicians for decades, and is a question of choice between two states in the presence of uncertainty. The problem so far, is considered open and there has been no agreed approach or framework for its analysis. In this paper we outline an elementary approach based on Cover's switching function that, in essence, makes a biased random choice where the bias is conditioned on the observed value of one of the states. We argue that the resulting symmetry breaking introduced by this process results in a gain counter to naive expectation. Finally, we discuss a number of open questions and new lines of enquiry that this discovery opens up.
\end{abstract}

Keywords: Random switching; two-envelope problem; exchange paradox; Parrondo's paradox; Brownian ratchets; symmetry breaking; game theory; volatility pumping; Cover's switching function.

\section{Introduction}

The two-envelope game is a hotly debated topic [1-12] that encapsulates the problem of decidability between two states in the face of uncertainty. This type of decision-theoretic scenario is of significance as it crops up, in different guises, in a number of fields ranging from physics and engineering to economics. Also, the twoenvelope problem attracts wide interest as it touches upon decision theory, game theory, and probability theory [13]. For ease of visualization, the problem is often entertainingly stated in game-theoretic form as follows. Imagine a player is presented with two envelopes, one containing $x$ dollars and the other $2 x$ dollars. The player randomly chooses an envelope and observes the amount inside. The question 
is, should the player keep that envelope or now swap it for the other one, in order to maximize payoff?

If the observed amount is $y \in\{x, 2 x\}$, then the other envelope must contain either $2 y$ or $\frac{y}{2}$ dollars. Thus by swapping, the player either moves ahead by a net $y$ dollars or drops back by $\frac{y}{2}$ dollars. Thus, it seems swapping is attractive as the amount the player possibly wins by is always more than the amount the player stands to lose. However, the conundrum is that exactly the same argument could have been used if the other envelope was selected first, hence we have an apparent paradox. As we shall see this can be resolved, and in this paper we will address the wider question that asks "is there a strategy one can adopt to maximize the payoff for an ensemble of two-envelope games?"

The two-envelope problem has some structural similarities to the Monty Hall problem [14, 15], Newcomb's paradox [16-18], and the St. Petersburg paradox [19], but should not be confused with these different games. As we shall see, in Sec. 3, the two-envelope problem leads to specifically different questions, has a unique mode of solution, and is further distinguishable in that it can be likened to ratcheting phenomena.

The origin of the problem traces back to 1930, when the Belgian mathematician Maurice Kraitchik described the essence of the conundrum, which he called the necktie paradox [20]. In this form, two players each have a necktie and they call an independent judge who takes the better necktie and awards it to the other player. A given player reasons that entering the game is advantageous: although there is a possible maximal loss of one necktie, the potential winning state is two neckties with one that is judged superior. However, the apparent paradox is that both players can follow the same reasoning, therefore how can the game be simultaneously advantageous to both players? In 1953, Kraitchik reframed this problem using two wallets containing money [21] and in 1982 Martin Gardner dubbed this the wallet game [22]. Independently, in 1953, the mathematician J. E. Littlewood described essentially an identical paradox, but based on playing cards, and attributed the game to the physicist Erwin Schrödinger [23]. The problem then appears in its present two-envelope form in 1988 [24].

It should be noted that variants of the two-envelope game do exist [25, 26], and in this paper we specifically analyze the case where the amount $x$ is unbounded, continuous valued, and that we have a large ensemble of independent games to play.

In the following section, we show the remarkable result that a switching strategy does exist that guarantees an expectation of positive payoff for multiple independent plays. The switching scheme we adopt is that when the player observes the amount in the first selected envelope, the player swaps to the second envelope with a probability that is a function of the observed amount. We call this switching policy Cover's switching function, after Tom Cover who first suggested the idea [27, 28]. The key is to select a Cover switching function, $P(y)$, which decreases as $y$ increases. This is because we want to make it less likely to switch envelopes, if the observed amount is a large sum of money. Conversely, we want the probability of switching 
to be high if the initial observed amount is small. Thus $P(y)$ is expected to be some monotonically decreasing function of the observed amount. As the analysis in Sec. 2 shows, this scheme in fact does work. In Sec. 3 we explain this physically in terms of a ratcheting effect due to symmetry breaking - i.e., if we leave the envelopes unopened the problem becomes symmetrical, but as soon as we open an envelope and switch according to $P(y)$ we break this symmetry.

\section{Analysis of the Two-Envelope Problem}

Suppose two envelopes contain continuous valued amounts $x$ and $2 x$, respectively. The player selects one at random, it is opened and the amount revealed. The player now has the opportunity to switch to the other envelope; and the question is should the player swap? Firstly, let us imagine the player does not open the envelope. The situation is entirely symmetrical and the average return is:

$$
R=\frac{1}{2}(x+2 x)=\frac{3}{2} x .
$$

Thus the player's expected return, $R=1.5 x$, no matter if the envelopes are switched or not. Now suppose the first envelope is opened and that the value observed is $y$ dollars. If the player now switches envelopes with a probability $P(y) \in[0,1]$, the average return for a particular $x$ is then:

$$
\begin{aligned}
R & =\frac{1}{2}[x(1-P(x))+2 x P(x)]+\frac{1}{2}[2 x(1-P(2 x))+x P(2 x)] \\
& =\frac{3}{2} x+\frac{1}{2} x[P(x)-P(2 x)] .
\end{aligned}
$$

Hence we clearly improve the return if $P(x)>P(2 x)$. The question is, what function do we to choose for $P(y)$ ? It appears that any monotonically decreasing function will improve the return. This result is quite general and does not presuppose a probability density function $p(x)$ for $x$.

If we now select a suitably monotonically decreasing function, for example $P(y)=\exp (-a y)$, this then leads to a question of optimization. How do we optimize the value of the coefficient $a$ in order to maximize the payoff $R$ ? The problem is that $a$ depends on the value of $x$, which is unknown. In the absence of any knowledge about the probability density function, $p(x)$, we cannot optimize $a$ as there is insufficient information.

However, let us now consider the case that, while $x$ is unknown, we know its probability density function (pdf) $p(x)$. For example, suppose the pdf follows an exponential distribution $p(x)=c \exp (-c x)$. The average return if the envelope is unopened is,

$$
\bar{R}=\int_{0}^{\infty} \frac{3}{2} x c e^{-c x} d x=\frac{3}{2 c},
$$

and by symmetry this holds regardless of whether there is no swap, always a swap, or a random swap. Now, in the case where we open an envelope, observe $y$, and 
randomly switch according the function $P(y)=\exp (-a y)$ we have:

$$
\begin{aligned}
\bar{R} & =\int_{0}^{\infty}\left[\frac{3}{2} x+\frac{1}{2} x\left(e^{-a x}-e^{-2 a x}\right)\right] c e^{-c x} d x \\
& =\frac{3}{2 c}+\frac{c}{2(c+a)^{2}}-\frac{c}{2(c+2 a)^{2}} .
\end{aligned}
$$

Differentiating to find the maximum, we have:

$$
\frac{d \bar{R}}{d a}=-\frac{c}{(c+a)^{3}}+\frac{2 c}{(c+2 a)^{3}}=0 .
$$

Solving the resulting cubic yields $a=0.35121 c$, and substituting this back into Eq. (4) yields the average return as,

$$
\bar{R}=\frac{1.6013}{c}
$$

Now let us suppose we do not select an arbitrary $P(y)$, but seek now to find the optimal switching function. What we require is to find the optimal function $P(y)$, which maximizes $\bar{R}$, for the pdf $p(x)$, with $P(y)$ constrained such that $P(y) \in[0,1]$, thus:

$$
\begin{aligned}
\bar{R} & =\int_{0}^{\infty}\left\{\frac{3}{2} x+\frac{1}{2} x[P(x)-P(2 x)]\right\} p(x) d x \\
& =\int_{0}^{\infty} \frac{3}{2} x p(x)+\left[\frac{1}{2} x p(x)-\frac{1}{8} x p(x / 2)\right] P(x) d x .
\end{aligned}
$$

This will be maximized if we choose $P(x)=1$ if $p(x)>0.25 p\left(\frac{x}{2}\right)$ and $P(x)=0$ otherwise. This result is general for any pdf $p(x)$, but requires knowledge of $p(x)$ in order to evaluate the inequality. To see how this might be carried out, for illustrative purposes, let us select our specific example of $p(x)=c \exp (-x)$. Therefore, we want $P(x)=1$ if $c e^{-c x}>0.25 c e^{-0.5 c x}$, which reduces to the following inequality:

$$
x<\frac{2 \ln (4)}{c} .
$$

Hence the average return will be:

$$
\begin{aligned}
\bar{R} & =\frac{3}{2 c}+\int_{x_{o} / 2}^{x_{o}} \frac{1}{2} x c e^{-c x} d x \\
& =\frac{1.6804}{c},
\end{aligned}
$$

where $x_{o}=\frac{2 \ln (4)}{c}$. Notice that, as required, the average return in Eq. (9) is indeed higher than that obtained in Eq. (6) before the optimization procedure. 


\section{The Two-Envelope Switching Process as a Ratchet}

As we have shown in Sec. 2, the use of a Cover switching function not only provides a good framework for handling the two-envelope problem but also remarkably provides a path for optimizing the player's payoff. Whilst, this is initially surprising, we now discuss the physical picture and indicate how the two-envelope process nicely accords with the mechanism of a discrete-time Brownian ratchet.

In game theory, Parrondo's principle is paradigmatic of a discrete-time ratchet and shows us that random switching between two losing games, in some circumstances, can result in a winning expectation [29-34]. A useful picture for understanding how this works, is to notice that the states in one of the games are set up such that there is detailed balance when the game is played in isolation, but when it is randomly mixed with another losing game the symmetry of the balance is broken resulting in different equilibrium probabilities that can be designed to then result in a winning game. This interaction between symmetry breaking and random behavior is a ratchet effect and, in the case of Parrondo's games, this connection to ratchets has been formally established via discretization of the Fokker-Planck equation [35-37].

Another interesting example is a new form of Parrondo's paradox called the Allison mixture [34]. Here we have two independent sequences of random digits that individually have an autocovariance $\rho=0$, and when we randomly mix the sequences the resulting new sequence can result in $\rho \neq 0$. The naive expectation is that a random mixture of sequences, individually possessing $\rho=0$, should also result in a final sequence with $\rho=0$ - however it can be shown $[38,39]$ that $\rho$ is only zero provided the sequence means $\mu_{1}=\mu_{2}$ or the switching transition probabilities $\alpha_{1}+\alpha_{2}=1$. If we break both these conditions, then we can legally produce a sequence with a $\rho \neq 0$. The idea is that if we break symmetry and impose both $\mu_{1} \neq \mu_{2}$ and $\alpha_{1}+\alpha_{2} \neq 1$, we lose detailed balance and end up with a ratcheting action that results in a final sequence with $\rho \neq 0$ [34].

In Parrondo's original games and the Allison mixture we see that when there is symmetry we have detailed balance in both cases, and the processes are then both time-reversible. When we then break symmetry both processes become timeirreversible, and we obtain an increase in some parameter that moves against our naive expectation. This is precisely what happens in the thermodynamic picture of the Feynman-Smoluchowski ratchet: at equilibrium there is detailed balance, timereversibility, and no net displacement; whereas out of equilibrium detailed balance is broken, with time-irreversibily leading to net displacement [34].

We can now appreciate that this is precisely the type of behavior we observe in the two-envelope problem. When the envelopes are closed, the situation is symmetrical and swapping envelopes is purely a time-reversible situation. However, as soon as we open an envelope, make an observation, and switch with $P(y)$ conditioned on the observed amount we have broken symmetry and made the process time-irreversible. Hence, we now see the physical picture that winning money on 
the two-envelope process appears to be consistent with the thermodynamic viewpoint if it is pictured as a ratchet and this now motivates future work to establish a formal link.

Finally, it is worth pointing out in hindsight from an information-theoretic viewpoint one should not be so surprised that the average return increases via performing a decision based on the observed amount $y$. This is because observing $y$ is a partial measurement of the whole system and has thereby reduced uncertainty and increased information. However it is nevertheless intriguing that average gain can increase without prior knowledge probability distribution of $p(x)$.

\section{Conclusion and Outlook}

In this paper we have introduced the Cover switching function that is a transition probability between two states, conditioned on the value of the initial state. We have shown that if this is chosen to be an arbitrarily monotonically decreasing function and adopted as a switching strategy, in the two-evelope game, we can calculate a net positive payoff for the player. We show this is independent of the probability distribution function (pdf) of the value of the initial state. Furthermore, in the case when a pdf is known, we have shown that we can optimize the strategy to maximize the payoff.

This work now motivates a number of interesting open questions for future exploration. We have analyzed a specific example with an exponential distribution, and this leads naturally to the question of researching how the payoff varies with different distributions. In particular, fat-tailed distributions as observed on the stock market would be of topical interest. This also leads to the question of exploring connections with Luenberger's method of volatility pumping on the stock market [40] and if a common mathematical framework can be developed.

It is known that processes that switch between two different distributions can be useful, for example, the statistical distribution of words in a human language can be modeled in this way [38]. This leads to the question of new forms of repeated two-envelope games where the amount $x$ is alternately selected from two different different distributions. Another possibility is that $x$ might be selected from $N$ possible distributions, and there remains the question of whether a form of central limit theorem can be established in this case. Moreover, it is known there exists a Gaussian decomposition theorem for arbitrary distributions [41]. Therefore, another line of enquiry would be to compare the two-envelope game based on one given distribution $p(x)$, with an ensemble of many games each played on the set of Gaussian components of $p(x)$.

Games of chance and strategy are of emerging interest in the fields of physics [42, 43] and econophysics [44] and a classical game of chance of this type can be transformed into a quantum game setting. This type of approach is furthering our understanding of quantum systems [45-47], and a biased quantum walk based on the two-envelope game would be a possible starting point. 
Finally, another interesting line of enquiry would be to recast the two-envelope switching process into the language of information theory - the relevant questions here are to ask are how many bits of information are inferred by observing the amount in the envelope, how does information entropy evolve during the switching process, and by analogy does it accord with thermodynamic models of entropy or run counter to expectation?

\section{References}

[1] R. Christiansen and J. Utts, Bayesian resolution of the 'exchange paradox', The American Statistician 46 (1992) 274-276.

[2] T. McGrew, D. Shier and H. Silverstein, The two-envelope paradox resolved, Analysis 57 (1997) 28-33.

[3] P. Castell and D. Batens, The two-envelope paradox: The infinite case, Analysis 54 (1994) 46-49.

[4] M. Clark and N. Shackel, The two-envelope paradox, Mind 109 (2000) 415-442.

[5] C. J. G. Meacham and J. Weisberg, Clark and Shackel on the two-envelope paradox, Mind 112 (2003) 685-689.

[6] C. J. Albers, B. P. Kooi and W. Schaafsma, Trying to solve the two-envelope problem, Synthese 145 (2005) 89-109.

[7] P. Rawling, Perspectives on a pair of envelopes, Theor. Decis. 43 (1997) 253-277.

[8] F. Jackson, P. Menzies and G. Oppy, The two-envelope paradox, Analysis 54 (1994) $43-45$.

[9] S. J. Brams and D. M. Kilgour, The box problem: To switch or not to switch, Math. Mag. 68 (1995) 27-34.

[10] R. A. Agnew, On the two-box paradox, Math. Mag. 7 (2004) 302-308.

[11] N. M. Blachman and D. M. Kilgour, Elusive optimality in the box problem, Math. Mag. 74 (2001) 171-181.

[12] F. Dietrich and C. List, The two-envelope paradox: An axiomatic approach, Mind 114 (2005) 239-248.

[13] B. Langtree, The classical and maximin versions of the two-envelope paradox, Australas. J. Logic 2 (2004) 30-43.

[14] M. vos Savant, Letters to the editor, The American Statitician 45 (1991) 347.

[15] A. P. Flitney and D. Abbott, Quantum version of the Monty Hall problem, Phys. Rev. A 65 (2002) Art. No. 062318.

[16] R. Nozick, Newcomb's problem and two principles of choice, in Essays in Honor of Carl G. Hempel, Vol. 115, ed. N. Rescher (Synthese Library, Dordrecht, 1969).

[17] D. H. Wolpert and G. Benford, What does Newcombs paradox teach us? http://ssrn.com/abstract $=1381295$.

[18] E. W. Piotrowski and J. Sładkowski, Quantum transmemetic intelligence, in Quantum Aspects of Life, Chap. 14, eds. D. Abbott, P. C. W. Davies and A. Pati (Imperial College Press, 2008), pp. 291-309.

[19] D. Bernoulli, Originally published in 1738, trans. L. Sommer exposition of a new theory on the measurement of risk, Econometrica 22(1) (1954) 22-36.

[20] M. Kraitchik, Le paradoxe des cravates, La Mathematique des Jeux (1930), p. 253.

[21] M. Kraitchik, Mathematical Recreations (Dover, 1953).

[22] M. Gardner, Aha! Gotcha: Paradoxes to Puzzle and Delight (W. H. Freeman, New York, 1982).

[23] B. Bollobás, Littlewood's Miscellany (Cambridge University Press, 1997). 
[24] S. Zabell, Loss and gain: The exchange paradox, in Bayesian Statistics, Proc. of the Third Valencia International Meeting, eds. J. M. Bernardo, M. H. DeGroot, D. V. Lindley and A. F. M. Smith (Clarendon Press, Oxford, 1988), pp. 233-236.

[25] B. Nalebuff, The other person's envelope is always greener, J. Econ. Perspect. 3 (1989) 171-181.

[26] R. S. Nickerson and R. Falk, The exchange paradox: Probabilistic and cognitive analysis of a psychological conundrum, Thinking and Reasoning 12 (2006) 181-213.

[27] T. Cover, Private Communication (Stanford University, 2003).

[28] M. D. McDonnell and D. Abbott, Randomized switching in the two-envelope problem, Proc. R. Soc. A (2009), doi:10.1098/rspa.2009.0312.

[29] G. P. Harmer and D. Abbott, Losing strategies can win by Parrondo's paradox, Nature 402 (1999) 864.

[30] G. P. Harmer and D. Abbott, Parrondo's paradox, Statistical Science 14 (1999) 206213.

[31] G. P. Harmer and D. Abbott, A review of Parrondo's paradox, Fluctuation and Noise Letters 2 (2002) R71-R107.

[32] B. Cleuren and C. Van den Broeck, Primary Parrondo paradox, Europhys. Lett. 67 (2004) 151-157.

[33] B. Cleuren and C. Van den Broeck, Optimizing strategies in the primary Parrondo paradox, Phys. Rev. E 70 (2004), Art. No. 067104.

[34] D. Abbott, Developments in Parrondo's paradox, in Applications of Nonlinear Dynamics, eds. V. P. Longhini and A. Palacios (Springer-Verlag, Berlin, 2009), pp. $307-321$.

[35] A. Allison and D. Abbott, The physical basis for Parrondo's games, Fluctuation and Noise Letters 2 (2002) L327-L341.

[36] R. Toral, P. Amengual and S. Mangioni, Parrondo's games as a discrete ratchet, Physica A 327 (2003) 105-110.

[37] P. Amengual, A. Allison, R. Toral and D. Abbott, Discrete-time ratchets, the FokkerPlanck equation and Parrondo's paradox, Proc. Royal Society Lond. A 460 (2004) 2269-2284.

[38] A. Allison, C. E. M. Pearce and D. Abbott, Finding keywords amongst noise: Automatic text classification without parsing, Proc. SPIE Noise and Stochastics in Complex Systems and Finance, Florence, Italy (2007).

[39] C. E. M. Pearce, A. Allison and D. Abbott, Perturbing singular systems and the correlating of uncorrelated random sequences, Proc. AIP International Conference on Numerical Analysis and Applied Mathematics, Corfu, Greece (2007).

[40] D. G. Luenberger, Investment Science (Oxford University Press, 1997).

[41] B. Anderson and J. Moore, Optimal Filtering (Prentice-Hall, Englewood Cliffs, N.J. 1979).

[42] D. Abbott, Overview: Unsolved problems of noise and fluctuations, Chaos 11 (2001) $526-538$.

[43] D. Abbott, P. C. W. Davies and C. R. Shalizi, Order from disorder: The role of noise in creative processes: A special issue on game theory and evolutionary processes overview. Fluctuation and Noise Letters 2 (2002) C1-C12.

[44] N. F. Johnson, P. Jeffries and P. M. Hui, Financial Market Complexity (Oxford University Press, 2003).

[45] C. F. Lee, N. F. Johnson, F. Rodriguez and L. Quiroga, Quantum coherence, correlated noise and Parrondo games, Fluctuation and Noise Letters 2 (2002) L293-L297.

[46] A. P. Flitney and D. Abbott, Quantum Parrondo games, Physica A 314 (2002) 35-42.

[47] D. A. Meyer and H. Blumer, Quantum Parrondo games: Biased and unbiased, Fluctuation and Noise Letters 2 (2002) L257-L262. 